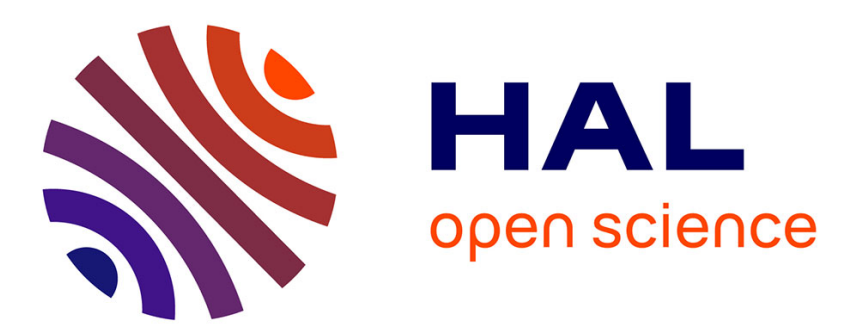

\title{
Faire la sourde oreille. Sociologie d'un conflit politique autour du bruit en ville
}

Elsa Lafaye de Micheaux

\section{To cite this version:}

Elsa Lafaye de Micheaux. Faire la sourde oreille. Sociologie d'un conflit politique autour du bruit en ville. Communications, 2012, 90, pp.109 - 129. 10.3406/comm.2012.2657 . halshs-03134974

\section{HAL Id: halshs-03134974 \\ https://shs.hal.science/halshs-03134974}

Submitted on 8 Feb 2021

HAL is a multi-disciplinary open access archive for the deposit and dissemination of scientific research documents, whether they are published or not. The documents may come from teaching and research institutions in France or abroad, or from public or private research centers.
L'archive ouverte pluridisciplinaire HAL, est destinée au dépôt et à la diffusion de documents scientifiques de niveau recherche, publiés ou non, émanant des établissements d'enseignement et de recherche français ou étrangers, des laboratoires publics ou privés. 


\section{Faire la sourde oreille. Sociologie d'un conflit politique autour du} bruit en ville

Elsa Lafaye de Micheaux

\section{Citer ce document / Cite this document :}

Lafaye de Micheaux Elsa. Faire la sourde oreille. Sociologie d'un conflit politique autour du bruit en ville. In: Communications, 90, 2012. Les bruits de la ville. pp. 109-129;

doi : https://doi.org/10.3406/comm.2012.2657

https://www.persee.fr/doc/comm_0588-8018_2012_num_90_1_2657

Fichier pdf généré le 30/11/2018 


\begin{abstract}
Noise pollution has rapidly emerged as a key public policy issue relevant for citizens everyday life and specifically revealed by the development of local-level participatory bodies such as neighborhood councils. Residents suffering from those nuisances interpret them either as marks of uncivil behaviour or as a weakening of the sense of community. The present article, focussing on the city of Angers and building on a participant observation carried out by the author holding the unusual position of town councillor, chronicles the mobilisation of several stakeholders (associations, tenants, elected officials, students) and its predictable failure. In fact, rather than being the instantiation of a "sociology of incivility", noise pollution designates a set of changes undergone by demographic, urban, and social structures.
\end{abstract}

\title{
Résumé
}

Le thème des nuisances sonores s'est rapidement imposé aux politiques publiques comme un enjeu du quotidien des habitants, notamment exprimé dans le cadre du développement d'instances participatives comme les conseils de quartier. Les habitants victimes de ces nuisances analysent ce phénomène en termes d'incivilités ou de détérioration du "vivre ensemble». Cet article retrace, dans le cas d'Angers et à partir d'une enquête de terrain participative où l'auteur occupait la position singulière d'élue municipale, la mobilisation des différents acteurs concernés (associations, riverains, élus, étudiants) et son échec prévisible. En effet, la question du bruit semble moins s'expliquer par une sociologie des incivilités que traduire des évolutions de la morphologie sociale angevine. 


\section{Faire la sourde oreille. Sociologie d'un conflit politique autour du bruit en ville}

\section{PARADOXES DE LA DOUCEUR ANGEVINE}

Angers est connue dans l'Ouest comme une jolie ville tranquille et à taille humaine; elle évoque pour la France entière la «douceur angevine» célébrée par Joachim du Bellay. Mais pour Julien Gracq le caractère de la ville est tout autre: "le génie d'Angers - s’il y a un génie du lieu - m’a toujours paru être celui du confinement: son site mesquin, choisi à l'écart du fleuve, fait songer à ces natures étriquées [...]. Cette respiration courte est presque immédiatement perceptible au promeneur étranger qui va au hasard des rues, surtout si ses pas se dirigent vers le quartier désert de la cathédrale, ses ruelles peuplées de chats dormeurs et de pots de géraniums, où à peine entrevoyait-on jadis, de loin en loin, flotter silencieusement la robe d'un prêtre : intrigues en vase clos, odeur caractéristique de renfermé social, microsociétés stagnantes et mesquinement conflictuelles ${ }^{1}{ }^{»}$. Gracq évoque aussi le lien organique avec la campagne, dont la ville, "pataude», garderait l'odeur, et un centre-ville propret, « appareil digestif discret de la rente foncière [...] aménagé pour les commodités douillettes d'une fin de vie cossue bien plutôt que pour le stress à l'américaine». Si la forme dont son enfance a fixé l'image s'est modifiée avec le temps, elle lui semblera à l'âge adulte presque faussée: «La ville a changé depuis mon enfance. Elle s'est animée; elle a troqué sa nonchalance presque paysanne pour une agitation de commande, sans grand substrat, qui y rend plus d'une fois la promenade ingrate $^{2}$.»

Prise entre la douceur et le confinement émollient, Angers offre aux habitants une réalité paradoxale : avec 147000 habitants, elle est aujourd'hui la dix-huitième ville de France. Troisième métropole de l'Ouest, après Nantes et Rennes, et devant Saint-Nazaire, elle se caractérise encore par un climat provincial très particulier, demeuré relativement paisible et 
protégé, au fort ancrage catholique. Elle se trouve régulièrement classée en tête des villes françaises pour sa qualité de vie ${ }^{3}$. Mais cette image un peu figée, selon laquelle, finalement, tout se passe bien et rien ne se passe vraiment, ne reflète plus la situation objective d'Angers. D'une part, avec $33 \%$ de logements sociaux et un revenu médian sous la moyenne nationale (hors Paris), elle n'est plus uniquement bourgeoise. D'autre part, sa démographie n’est plus seulement caractérisée par la forte représentation des familles nombreuses : elle connaît depuis quinze ans une très nette croissance de la part des étudiants dans la population totale.

Le centre-ville est le quartier le plus marqué par cette évolution de la morphologie sociale et des usages de la cité. Les bars, restaurants et autres établissements de nuit s'y concentrent et attirent sur un périmètre particulièrement exigu une population festive (ou de fêtards, c'est selon) qui se déploie de plus en plus dans la rue. Ainsi la sociabilité estudiantine se déroule-t-elle largement sous les fenêtres des riverains, ce qui crée des problèmes de voisinage, causés par les nuisances sonores nocturnes. En conséquence, les habitants se sont exprimés et se sont mobilisés sur ce thème. Face à ces vives réactions, la mairie, alors même qu'un travail de longue date était mené par les services techniques sur le bruit ${ }^{4}$, a cherché de son côté à ne pas avoir à prendre en compte cette demande des habitants, faisant semble-t-il la «sourde oreille». L'auteur de cet article s'est trouvée au cœur du conflit angevin sur le bruit entre 2008 et 2010 à plus d'un titre: en tant qu'élue municipale du centre-ville, chargée d'opérer le lien entre les habitants et la politique municipale ${ }^{5}$, elle a été confrontée au processus complexe de mise en politique du bruit intempestif la nuit. Par ailleurs maître de conférences à l'Université publique, elle avait aussi une connaissance directe d'une partie de la population mise en cause.

C'est de ce conflit à plusieurs entrées, liées au fait que l'objet «bruit» catalyse lui-même de très nombreux enjeux - psychologiques, sociaux, économiques, politiques et symboliques ou techniques -, que traite cette étude. Elle vise à montrer comment, à Angers, le bruit en ville et plus précisément les nuisances sonores nocturnes rencontrent des difficultés pour être inscrits à l'agenda politique, car la mairie ne le souhaite pas véritablement. Et aussi comment cette question du bruit est un excellent révélateur des tensions visibles ou latentes liées aux conflits engendrés par les évolutions de la morphologie sociale sur un territoire restreint.

Inspiré par des travaux ethnographiques aux marges de la sociologie interactionniste en France, et en particulier de l'auto-ethnographie goffmanienne, cet article propose de revenir a posteriori sur ce conflit entre les habitants et la municipalité autour du bruit. Outre la description ethnographique du conflit vécu de l'intérieur, une perspective sociologique en 
termes de morphologie sociale ${ }^{6}$ sera adoptée pour expliquer le malentendu profond existant entre certains habitants. Le matériau réside dans la série des réunions ou rencontres, en public et en comité restreint, sur le sujet. En effet, au cours de ces réunions l'auteur prenait des notes, à dire vrai moins pour préparer un travail académique que pour se donner une contenance. C'était aussi une manière de montrer qu'elle accordait de la considération à la discussion et à la parole de ses interlocuteurs. Les courriers échangés au sein de la mairie sur le sujet et les courriers reçus des habitants, les courriers rédigés en réponse et les articles de journaux (le Courrier de l'Ouest, inlassable opposant à l'équipe en place, et Ouest France ${ }^{7}$ ) complètent le corpus. Un sentiment de désajustement profond vis-à-vis du rôle d'élue locale l'a conduite à démissionner de son mandat au bout de deux ans. En 2011, la distance permise par le recul et la fin du devoir de réserve l'autorisent à prendre pour objet le problème du bruit la nuit aux alentours des bars et des boîtes de nuit, tel que les habitants l'ont porté à la connaissance de la mairie.

\section{LA DIFFICILE MISE EN POLITIQUE DU BRUIT}

Le processus de transformation du problème du bruit la nuit en ville (causé par une jeunesse noctambule et festive) en décision politique est aussi complexe que délicat; il n'est pas dit que son aboutissement concorde avec les attentes de ceux qui l'ont exprimé, ni même en vérité qu'il aboutisse. Il serait faux de prétendre qu'à Angers l'adjointe au centre-ville y était bien préparée ${ }^{8}$. Partie d'un a priori sceptique sur le phénomène, convaincue que ceux qui se plaignaient étaient les «mauvais coucheurs » d'un centre-ville privilégié, j’ai peu à peu saisi la souffrance vécue par les habitants : directement engendrée par une fatigue récurrente, elle est aussi alimentée par l'angoisse du surgissement du bruit la nuit et par la violence qui consiste à se sentir nié ("c'est comme si on n’existait pas»). Enfin, je n'ai pas immédiatement pris la mesure de la profondeur et de l'étendue des enjeux engagés dans ces conflits, aux acteurs à la fois individuels et collectifs. Mon propre jugement sur le sujet a ainsi beaucoup évolué au fur et à mesure des rencontres et des événements.

Il est difficile de retracer la chronologie de la prise en considération politique du problème du bruit à Angers, notamment parce que celle-ci n'a pas directement eu lieu dans les termes que les habitants auraient voulu lui donner, et parce que le sens de l'histoire dans ce domaine est, aujourd'hui encore, tout sauf clair. Une présentation en termes de conflit ${ }^{9}$, avec son point d'orgue, où l'on éclairera le positionnement des différents acteurs en 
présence, sera sans doute plus pertinente, au risque de rendre artificiellement intelligible et ordonné un processus qui l'a très peu été pour ses protagonistes. On s'attachera à relater comment la question du bruit a été placée sur le devant de la scène par les habitants, de manière isolée ou plus organisée, et à décrire la manière dont la mairie, alors même que l'élue au centre-ville aurait souhaité donner suite à la demande, a toujours semblé chercher à éviter le sujet.

\section{Mobilisés pour une prise en compte politique du bruit.}

La question du bruit en ville est d'abord issue d'une requête publiquement exprimée par des habitants auprès de la mairie. Elle s'est étoffée à travers la mobilisation du conseil consultatif de quartier (CCQ) du centreville sur ce sujet, dans le droit-fil d'un travail entamé lors du précédent mandat et destiné à élaborer à la demande du maire une "Charte de bonne conduite». Celle-ci avait été conçue en réaction à ce qui était vu comme une forme d'incivilité des étudiants et rappelait des règles communes de la vie urbaine. Jugée assez répressive et autoritaire par la municipalité, elle résultait au contraire, selon les membres du CCQ, d'une volonté de tenir compte de tous les aspects de la question et cherchait à tout prix à ne pas dresser les uns contre les autres : locataires/propriétaires, jeunes/vieux, étudiants/actifs, Angevins/étrangers à la ville. Bien que le CCQ se fût appliqué à répondre à cette saisine du maire ${ }^{10}$, il ne lui avait pas échappé que celle-ci ressemblait fort à une manœuvre dilatoire pour ne pas faire autre chose (sous-entendu de plus efficace). La Charte n'était cependant pas allée au bout de sa vocation puisque sa distribution dans le quartier (33000 habitants) en était restée à une échelle confidentielle. Mais, pour ses auteurs, elle avait néanmoins porté quelques fruits, car « du côté de l'École supérieure d'agriculture, il n'y a plus eu ensuite de dégradations (rétroviseurs arrachés) et de cris dans la nuit». À la mise en place du nouveau CCQ du centre-ville au printemps 2009, il était manifeste que ce sujet constituait pour certains habitants la seule raison de participer au conseil de quartier, vu comme un espace de parole collective à investir : «je suis venue au CCQ pour le bruit. C'est la raison pour laquelle nous nous sommes inscrites».

Mais à côté d'un conseil de quartier dont une partie était très motivée par ce sujet, des témoignages individuels et des doléances privées se sont manifestés très tôt après les élections : par leurs courriers, demandes de rendezvous, lors d'échanges en marge des inaugurations ou autres manifestations publiques, des habitants ont souligné auprès de la nouvelle équipe les problèmes rencontrés la nuit dans certaines rues du centre. Adressés au maire, 
via son cabinet, ou à moi-même suite à la présentation de ma fonction dans le journal municipal local, ces courriers attestaient de hurlements, de vociférations, de concours de cris, de foules déchaînées, etc. L'alcool semblait très souvent impliqué dans la licence constatée, servi parfois dans des verres en plastique et bu debout directement dans la rue. Autre élément venant compléter le tableau décrit: les attroupements incontrôlables sur la voie publique, y compris dans des petites voies étroites ouvertes à la circulation où l'accident est toujours à craindre. Enfin, certaines heures où les boîtes de nuit sont tenues de fermer mais où les bars ne sont pas encore ouverts paraissaient propices à ces déambulations très sonores. Les afters, bars aux autorisations spécifiques, ouverts après l'heure de fermeture officielle, étaient alors pris d'assaut et les logements situés autour subissaient un bruit parfois très important. Dans les rues les plus exposées, les traitements individuels du problème par calmants ou somnifères étaient souvent évoqués. L'angoisse de se coucher avec l'idée que l'on va peut-être être réveillé en sursaut par des cris venus de la rue m'a été plusieurs fois exprimée : ainsi, pour certains, c'est moins le bruit lui-même que la peur de sa survenance qui est source de mal-être.

Enfin, la plus grosse association d'habitants du centre-ville, l'association Thiers-Boisnet, qui porte le nom de rues phares de la vie nocturne angevine, intervenait également dans le débat en orchestrant au niveau du quartier la grogne à l'encontre du maire. Dirigée dans un objectif largement politique par un proche collaborateur du principal opposant du maire, cette association alimentait le mécontentement vis-à-vis de la politique de la Ville sur tous les sujets imaginables: le tramway, le plan local d'urbanisme, le bruit, les déchets, etc. Elle m'avait invitée à assister à sa première assemblée générale post-élections (en janvier 2009) pour que j’y expose les actions de la mairie sur le thème du bruit et pour que j'entende les remarques et critiques de ses membres. La salle municipale était comble, et j'y avais longuement et patiemment présenté le dispositif en faveur des soirées étudiantes, dit «S'amuser sans abuser», mis en place par la mission Jeunesse auprès des associations étudiantes en lien avec les établissements ${ }^{11}$. C'est là que j'ai entendu rapporter la célèbre phrase (réelle ou apocryphe) du maire au public du centre-ville : "Si vous n'aimez pas le bruit, allez vivre à la campagne ! » Très présente au sein du CCQ, où elle occupe des positions dans tous les secteurs, l'association Thiers-Boisnet a milité activement pour qu'un groupe de travail spécifique se constitue à nouveau sur le thème des nuisances nocturnes.

Un autre type d'accusation portée à l'encontre des pouvoirs publics par des personnes rencontrées est de chercher à favoriser le commerce en centre-ville, au détriment de la vie de ses habitants : "On est une entrave. Les gens qui habitent dans le centre-ville, on gêne, on compte moins que 
l'animation [de Noël], que les magasins!» Une «Charte des bars et établissements de nuit » avait été rédigée au cours du mandat précédent pour rappeler ces professions à leurs obligations, mais la commission des bars et établissements de nuit qui l'avait établie et restait chargée de la faire circuler n’a jamais été vue comme très sérieuse : les liens personnels, voire les connivences, entre les membres de cette commission et les bars visés étaient dénoncés, notamment par d'autres commerçants. En effet, la présence en son sein de l'adjoint au commerce, bon vivant, ancien charcutier et ancien président de la chambre des métiers, ôtait quelque peu de sa vertu au dispositif et entamait la crédibilité de ses actions. Des habitants ont ainsi mentionné des bars «qui auraient dû se prendre une fermeture administrative. On sait pourquoi ils n'ont rien eu. Ce n'est pas possible avec tout ce qu'ils servent ! À des mineurs, en plus aussi ! À des jeunes qui sont déjà saouls ! ».

Finalement, c'est un article paru début juin 2009 dans le journal local, relatant un «cri de détresse adressé au maire », qui a porté le débat sur la scène publique et constitue le point d'orgue de cette mobilisation ${ }^{12}$. Oscillant entre colère et épuisement, un couple interviewé témoignait de ses nuits impossibles dans un appartement situé en plein centre: "nous sommes à bout: nous entendez-vous? ». Cet article, publié vraisemblablement avec gourmandise par le journal d'opposition ${ }^{13}$, avait choisi de placer (et déplacer, en fait) cette action au cour de la revendication d' « un débat sur la nécessité d'une présence accrue de politiques et médiateurs sur le terrain ». Ce glissement s'est opéré indépendamment de ce qu'en attendaient confusément les habitants interrogés, "pas forcément partisans d'une politique répressive ». En effet, alors que la répression était supposée être le désir des habitants, selon le journal local, des attentes plus simples et moins disciplinaires s'exprimaient dans leurs témoignages: "Nous, ce qu'on veut, c'est qu'il se passe des choses, c'est voir qu'il y a des actions mises en place et sentir une amélioration. »

Cet article de presse sur le bruit en centre-ville a déclenché un grand nombre de courriers, pour certains publiés, ce qui a fini par former un feuilleton de plusieurs semaines. Au même moment, les étudiants étaient clairement incriminés, dont ceux de l'école de commerce locale : celle-ci a cru bon de faire paraître un démenti, à son tour vivement remis en question, y compris par un courrier du maire. Dans le même temps, les festivités liées aux soirées de fin d'année des écoles et cursus universitaires, le temps estival ou la Fête de la musique ont démultiplié les occurrences du bruit.

Enfin, le «cri de détresse » mettait en évidence l'exaspération engendrée par le bruit. Comme le disait le mari interviewé, le « seuil de tolérance » des habitants était dépassé : «si je ne dormais pas avec des boules Quies, il se 
serait passé quelque chose ». De même, une «petite dame » racontait début juin au CCQ que, excédée, elle avait braqué son tuyau d'arrosage sur des groupes en pleine nuit. De plus en plus d'habitants menaçaient de "péter les plombs », de ne pas réussir à contenir une fureur grandissante, voire d'aller se charger eux-mêmes de «les » faire taire : "j’ai jusque-là réussi à calmer mon mari, à l'empêcher de faire une grave bêtise, mais je ne suis pas sûre d'y arriver si ça continue comme ça ». De telles déclarations lors des appels pour tapage nocturne semblaient d'ailleurs le meilleur moyen d'obtenir l'arrivée rapide de la police sur les lieux.

\section{Les résistances de la mairie: bruit intolérable ou intolérance au bruit?}

La réaction de la mairie face au courrier est symptomatique de l'ambivalence qui marque l'ensemble de ses actes dans ce domaine du bruit en ville. D’une part, la prise en charge par le conseil de quartier a été immédiate et la mobilisation de l'élue de quartier rapide, mais, d'autre part, le maire et son cabinet restaient circonspects et ont temporisé. En effet, deux jours après la publication de l'article, le futur père de famille est arrivé hors de lui au CCQ. Amené là par une habitante membre du CCQ (qui avait pris contact avec lui suite à l'article), il venait, très agressif, pour rallier le groupe à sa cause ; il menaçait aussi de diffuser des vidéos prises dans la rue ou de faire une grève de la faim si rien n'était fait pour arranger les choses. Les habitants réunis sont parvenus à canaliser sa colère et lui ont proposé de travailler, avec eux, sur le projet d'un site internet du quartier (il était webmaster), espace d'échanges et de dialogues sur la vie en centre-ville. Le même soir, le conseiller municipal PS, assisté de la responsable administrative du pôle territorial de centre-ville, expliquait en avant-première aux habitants la dernière initiative prise par la mairie pour améliorer la situation : sur le modèle d'un dispositif nantais, ce projet dit des «veilleurs de soirée » devait développer une prévention de proximité contre l'alcoolisme auprès de jeunes noctambules (dans les rues les plus passantes), avec l'espoir de réduire les cris et autres débordements. La fonctionnaire présente, responsable du pôle territorial, a choisi d'écrire immédiatement à sa hiérarchie et au cabinet du maire pour « alerter sur la montée en puissance de la colère des habitants de l'hyper-centre » et pour suggérer l'urgence de les contacter. Dès les jours suivants je recevais le couple dans mon bureau pour entendre sa situation et le présentais ensuite à l'adjoint à la prévention et la sécurité, qui lui exposait les mesures prises, mais aussi la nécessité qu’il dépose plainte afin que sa demande soit prise en compte. 
"Ça sent la manipulation», analysait le directeur de cabinet, ce qui, lorsqu' on connaît les conditions de production du «témoignage », pouvait ne pas être faux. Ainsi la réponse très attendue a-t-elle mis beaucoup plus de temps à leur parvenir : le courrier officiel n'a quitté le cabinet que le 23 juin. Entre-temps, les échanges par journal interposé avaient été très nombreux - plus tard, ce couple admettra avoir été dépassé par l'ampleur de ces réactions et par le rôle de porte-parole qu'on a rapidement attendu de lui, que ce soit de la part du quotidien local ou des habitants rencontrés.

Pour les fonctionnaires municipaux ayant déjà travaillé lors du précédent mandat sur ce sujet dans le cadre de la participation des habitants, la question de fond était en réalité de savoir si le bruit était intolérable ou si ce n'étaient pas plutôt les gens du centre-ville qui étaient intolérants. Cette mise en doute, exprimée au moment où je prenais mes fonctions, au printemps 2008, se justifiait d'autant plus que les liens de confiance entre la mairie et le CCQ du centre-ville s'étaient fortement dégradés. Son ancien président, conseiller municipal de la majorité en place, avait commencé à soutenir clairement le candidat de l'opposition et à mettre en cause publiquement l'action municipale socialiste. Le cabinet du maire et les anciens responsables de la première expérience participative de la ville étaient d'autre part sévères à l'égard d'un CCQ jugé exigeant et compliqué par rapport aux conseils des autres quartiers. Au début de la présentation qu'ils m'ont faite du quartier, ils ont tenu un discours selon lequel « dans le centre-ville, les gens ne supportent pas d'entendre les autres, sont intolérants, se plaignent beaucoup de ça car ils n'ont pas d'autres problèmes plus lourds à régler [population plus favorisée]». Cette analyse reposait sur le fait que le centre-ville, où les cadres sont surreprésentés, vote à droite.

\section{Bruit et technologies du pouvoir.}

Dès notre première entrevue au moment de la constitution de la liste municipale, le maire avait pourtant évoqué la question des nuisances sonores comme l'une de celles auxquelles il faudrait que je sois particulièrement sensible sur mon quartier dans le cadre des fonctions qu'il me déléguait. Pionnier en France dans ce domaine en tant qu'ancien membre du Conseil national du bruit, et bon spécialiste du sujet, il semblait paradoxalement ne pas souhaiter y porter une attention plus précise. «Je n’ai pas de baguette magique, expliquait-il, il n'y en a pas sur ce sujet»; or inviter les habitants à en parler pouvait signifier que nous aurions une solution à leur offrir. Le sujet étant complexe, diffus et à déterminants multiples, aucune 
action publique simple et rapide n'était à même d'en venir à bout. C'est donc pour ne pas susciter de faux espoirs qu'il ne fallait pas l'aborder. Le cabinet, pour des raisons plus prosaïques, suivait cette ligne : une réunion dont l'issue serait à tout coup une confrontation et une pluie de reproches, une «soirée tomates», était à éviter absolument.

Ainsi, au second semestre 2008, lorsqu'il s'agit de reformer le conseil de quartier (CCQ 2) du centre-ville sur des règles légèrement rénovées et avec de nouveaux membres, suite aux Assises angevines de la participation, l'objectif qui me fut assigné, en tant qu'élue et coprésidente du conseil consultatif de quartier, fut d'essayer de « faire plutôt travailler sur le vivre ensemble, l'accueil des nouveaux habitants en centre-ville», et d'éviter de laisser les membres du CCQ se polariser sur le bruit. Lorsque ce problème arrivait dans la discussion en réunion, c'était donc au milieu de nombreux autres sujets sur lesquels les citoyens étaient amenés à réfléchir et à participer : travaux du tramway, aménagement de place, emplacements sécurisés pour des vélos, auto-partage, projet du nouveau quartier Gare + (ZAC), etc. C'est par la gestion de l'ordre du jour et la limite posée aux digressions que l'on pouvait laisser de côté ou réserver à d'autres contextes le travail sur les nuisances sonores. Dans ce quartier, les membres du conseil s'étaient regroupés entre trois thèmes : stationnement et déplacements, urbanisme ou «cadre de vie». Ce dernier groupe aurait dû par définition concerner les «nuisances sonores » parmi d'autres sujets. Mais les saisines, qui fixent le tempo de l'activité des CCQ, lui confiaient plutôt le soin de préparer et d'organiser la soirée d'accueil des nouveaux habitants du quartier, ou de concevoir le site internet (sorte de blog) du centre-ville. Ces saisines arrivaient au fil d'un calendrier défini par la mairie elle-même avec toujours un certain caractère d'urgence. Le travail d'animation et de contrôle de ce groupe de travail «cadre de vie», qui contenait le sujet délicat des nuisances nocturnes, avait été visé par le cabinet: son animation avait été confiée au jeune élu socialiste, étudiant en droit et conseiller municipal délégué à la jeunesse parfaitement rompu à l'exercice politique - tout benjamin de l'équipe qu'il fût. Mon inexpérience, mon empathie avec les habitants et leur souffrance, mon peu de goût pour les jeux politiciens ne pouvaient qu'inquiéter un cabinet habitué à la difficulté de l'exercice et aux manœuvres de l'opposition ${ }^{14}$. D'une manière générale, on m'a interdit jusqu'à la fin 2009 de conduire une réunion publique sur le sujet du bruit, et toutes mes interventions dans le domaine étaient précisément encadrées. Ainsi, l'animation du groupe spécifiquement dédié au sujet m’échappait. Mon intervention sur les nuisances nocturnes à l'invitation de l'association de quartier était contrôlée par le biais d'un powerpoint fourni par la mairie et dont le contenu m'était imposé. À défaut de pouvoir tenir une réunion publique dont je voyais très 
bien la fonction symbolique, voire cathartique, on me demandait de privilégier les interventions dans le contexte de relations individuelles : chez un habitant, dans le local de ma permanence d'élue, au rez-de-chaussée de la mairie, ou dans mon bureau. La distinction que pose Michel Foucault entre la discipline et la sécurité me semble éclairer ce conflit ${ }^{15}$ : là où les habitants présents dans les CCQ ou dans l'association Thiers-Boisnet attendaient ou espéraient confusément une réponse de type disciplinaire reposant sur l'obligation et la contrainte à partir d'une sorte d'idéal de vertus civiques partagées que l'on pourrait opposer aux incivilités, la municipalité interviendrait selon un régime de sécurité. La sécurité est entendue par Michel Foucault comme devant être atteinte d'une manière fondamentalement libérale: cette technologie du pouvoir reposerait non sur l'idéal mais sur la réalité, ses structures contingentes et ses régularités statistiques. L'action politique adopterait alors un point de vue très large sur le sujet tout en cherchant à manœuvrer à partir de plusieurs canaux d'intervention et d'incitation un réel laissé à lui-même pour l'essentiel, afin d'arriver à des fins de bien-être de la population. En suggérant à la mairie de répondre à la demande des habitants, je l'obligeais en fait à intégrer un régime d'action politique dont elle est, en tout état de cause, radicalement éloignée dans son fonctionnement même. C'est donc à cela aussi qu'elle se refusait en faisant, apparemment, la sourde oreille.

Face aux courriers sur le thème, alors que les doléances se multipliaient, y compris parfois en public, le cabinet du maire personnalisait les plaignants. Cette stratégie qui consiste à renvoyer à leurs intérêts privés des acteurs qui se font entendre dans la sphère publique peut sembler contradictoire avec l'esprit de la démocratie participative dans laquelle je devais m'inscrire. Elle cherchait sans doute avant tout à assurer la protection d'élus placés dans un cadre de relations aux habitants plus exposé qu'auparavant par les nouveaux dispositifs démocratiques. Ainsi tous les plaignants (par lettre, téléphone, intervention publique intempestive en réunion) étaient-ils repérés et catalogués, afin de désamorcer le caractère déstabilisant de leur remise en cause de l'action du maire, le plus souvent visée par ces courriers. Dès qu’une nouvelle personne avait été étiquetée, on m'en informait très vite pour m'aider à la repérer parmi mes interlocuteurs, afin de m’en méfier (ce qui se traduisait par le fait d'éviter de lui donner la parole en public) mais aussi de m'en protéger. Certains étaient présentés comme des proches de Béchu (le premier opposant politique au maire), en particulier les membres de l'association de quartier ; autrement dit, s'ils se plaignaient du bruit, c'était une manœuvre politique pour affaiblir le maire. D'autres utiliseraient ce vecteur pour régler leurs comptes avec la mairie, comme cette vieille dame donnant sur une venelle transversale devenue le déversoir (excréments, vomi, amoncellement de bouteilles 
cassées) de la principale rue des bars de la ville - bars dont les toilettes ne sont pas à la mesure du nombre des consommateurs servis. Vivant au rezde-chaussée, ses fenêtres, ainsi que celles d'autres personnes habitant dans le même passage public, donnaient directement sur les buveurs qui se soulageaient. Lorsqu'elle écrivait ou téléphonait à la mairie, ce qu’elle faisait sans retenue ayant été elle-même employée municipale pendant des décennies, ses messages étaient interprétés comme animés par l’amertume d'une situation professionnelle insatisfaisante. Une habitante à bout de nerfs multipliait les courriers et les appels? Elle et son mari se vengeaient sur la mairie d'un restaurant peu florissant dans une rue où les autres restaurateurs font recette et où les bars tournent à plein. Rencontrée dans son restaurant à plusieurs reprises, cette personne m'a pourtant semblée crédible dans ses revendications. Indépendamment des griefs personnels qu'elle pouvait entretenir vis-à-vis de la mairie (non évoqués en ma présence), elle a surtout mis en avant des éléments factuels de forte dégradation de l'environnement quotidien et manifesté des signes de grande souffrance.

Ce refus d'entendre les habitants exprimer leur souffrance et leur désarroi liés au bruit en ville la nuit, et de prendre en compte leur sentiment d'être abandonnés à leur sort, peut sembler d'autant plus paradoxal que, dans les faits, on ne peut pas reprocher au maire de "ne rien faire ». Au contraire, il existe un certain nombre de dispositifs municipaux anciens et nouveaux pour tenter de lutter contre les «nuisances sonores » à Angers. D’une part, dès la fin du mandat précédent, la Ville a élaboré avec les associations étudiantes la charte «S'amuser sans abuser» déjà évoquée : signée par la plupart d'entre elles après chaque rentrée universitaire, elle a pour vocation de sensibiliser les membres des bureaux des étudiants aux risques de l'alcoolisation abusive et à leur responsabilité en cas d'accident. Parallèlement, la commission des bars et établissements de nuit était mise en place. D'autre part, afin de favoriser la tranquillité publique en centreville, des médiateurs de jour ont été nommés à destination des SDF pour les empêcher d'importuner les passants, et surtout les clients du nouveau centre commercial «Fleur d'Eau », issu de très lourds investissements privés d'autant plus stratégiques que succédant à une série d'échecs à ce même endroit. Enfin, le nouveau mandat a été marqué par la création, longtemps gardée secrète, des "Noctambus», équipes d'étudiants faisant de la prévention auprès de leurs pairs in situ la nuit, sur le modèle nantais. Ce dispositif a été mis en place à l'automne 2009, après les événements médiatiques de juin. Mais la campagne de communication que j'avais demandée, en réponse à l'affirmation lancinante « le maire ne fait rien pour nous, le maire s'en fout », sur l'ensemble des mesures prises n'a jamais été préparée. 
La cristallisation du conflit à l'occasion des publications de témoignages du Courrier de l'Ouest alimentés en direct par les éruptions de nuisances nocturnes au début des beaux jours a été suivie d'une accalmie durant l'été. À l'automne 2009, le conflit entre les habitants et la mairie a connu un épilogue provisoire (du moins dans le cadre de mon mandat), épilogue qui s'est déroulé en deux temps, ouvrant pour finir des occasions jusquelà rigoureusement condamnées. Premièrement, sous la pression des habitants présents au CCQ, cette instance participative a obtenu du maire en septembre l'autorisation de reprendre son travail sur le sujet. J'avais alors proposé d'organiser sur le thème une soirée animée par la directrice du CIDB (Centre d'information et de documentation sur le bruit) où des élus municipaux des métropoles voisines de Rennes et Nantes auraient été invités pour parler de ce problème dans leur cité et de la manière dont ils l'envisageaient. Les échanges avec la directrice du CIDB, elle-même proche du maire, avaient été très nombreux, car elle avait vite compris que le cabinet et moi-même formulions des demandes pas tout à fait convergentes. Je devais aller suivre une formation d'élue trois jours à Paris au CIDB. Les membres du CCQ devaient quant à eux préparer la réunion publique (qui ne devait pas s’intituler ainsi mais plutôt « réunion d'information ») en parlant autour d'eux.

Puis, de manière subite car faisant suite à une nuit où les forces de police se sont senties dépassées par la taille des attroupements sur la voie publique, une réunion a eu lieu au plus haut sommet sur la question du bruit à Angers. Cette réunion s'est tenue avec tous les responsables des services publics concernés: la préfecture (le directeur de cabinet du préfet), le commissaire de police, le procureur de la République et la mairie, représentée par l'élu à la sécurité et la prévention et son directeur de service. Cette première réunion visait à échanger sur les diagnostics du problème et à envisager des mesures visibles, rapides, qui frapperaient les esprits et poseraient une limite symbolique aux actions incontrôlées - la présence du procureur a donné soudain une tonalité très disciplinaire au problème. À partir de ce moment, la question du bruit devenu insupportable en centre-ville a commencé à toucher plusieurs adjoints, là où elle mobilisait de longue date leurs directions et services. En interne, une réunion a ensuite rassemblé tous ces acteurs (élus et services) pour un tour de table sur les dispositifs en place. Mais le scepticisme a dominé la discussion: de toute façon le résultat serait décevant, voire coûteux politiquement, sachant qu'a priori ces habitants votaient à droite. Ce sentiment partagé explique sans doute rétrospectivement la très faible mobilisation des élus sur le sujet au début du nouveau mandat. Néanmoins, dès lors qu'il est devenu une priorité de l'agenda municipal, je me suis trouvée « oubliée » de la liste des invités de la réunion avec la préfecture et le 
procureur et j’ai dû insister, arguant de ma légitimité d'élue de quartier, pour y siéger. Ma marginalisation signifiait qu'on ne tiendrait pas compte de la lecture des habitants pour construire une réponse au problème qui resterait abordé d'abord sous un angle technique, ensuite en imitant simplement des pratiques retenues par les villes voisines (Rennes, Nantes).

C' 'est cette lecture du bruit par les habitants eux-mêmes, mais aussi les éléments d'explication passés sous silence dans les interprétations majoritaires des acteurs, que la suite de cet article explore.

\section{LE SENS DU BRUIT}

Il semblerait que le décret d'application de 2006 étendant la loi Évin sur l'interdiction de fumer aux cafés et restaurants ait contribué à rendre le problème du bruit la nuit particulièrement intolérable. Les habitants qui s'en plaignent mettent d'abord en cause les étudiants. À Angers, la population estudiantine est essentiellement composée de jeunes ${ }^{16}$ d'origine le plus souvent éloignée d'Angers, dans les campagnes environnantes, voire dans les villes moyennes des départements alentour (Mayenne, Sarthe, Vendée) ${ }^{17}$. Nombreux à quitter Angers après les cours du vendredi, ils ont progressivement institutionnalisé le jeudi soir comme le soir où s'organisent les soirées étudiantes. À tel point que les cours du vendredi matin sont, du point de vue des enseignants, des créneaux à éviter. Ainsi, entre le jeudi soir, où les étudiants profitent d'une sociabilité urbaine nouvelle avant de «rentrer à la maison », et les soirées du week-end qui suit, les nuits du centre-ville sont mouvementées, au grand dam, et parfois pour la plus grande souffrance, des habitants plus anciens de ce quartier.

\section{Mépris ou mauvaise éducation?}

La question du sens à conférer à ces «nouveaux» comportements est extrêmement compliquée et renvoyait, dans ma position d'élue au croisement des positions et des discours, à la nécessité qui aurait dû guider mon action politique d'assigner une place dans le quartier à chacun (jeunes, étudiants, actifs, habitants de longue date, vieux), et de manipuler les symboles communs.

Avec le temps, il m’est apparu qu'en réalité c'était aussi et surtout ce qui accompagne le bruit qui n’était pas supporté. Ainsi ai-je vu mises en avant, dans les réunions ou rencontres relatives aux nuisances sonores, les thématiques suivantes : 


\section{Elsa Lafaye de Micheaux}

- les déchets: «on a le problème des bouteilles dans la rue; des poubelles laissées tout le week-end par les étudiants». Cela m'a amenée à demander une rencontre avec le service de la propreté qui fait les rondes dans le centre-ville, pour réclamer qu'il soit porté une attention particulière à des lieux très salis. Or les passages y étaient déjà plus fréquents et plus approfondis que dans les autres quartiers : "c’est parce que c'est la rue du maire ». Selon ce service, les éboueurs étaient eux-mêmes confrontés aux comportements incontrôlables des étudiants en goguette: «le problème c'est qu'ils font n'importe quoi à 5 heures du matin quand on passe: ils grimpent sur les containers, se mettent derrière la benne qui recule ».

- le vomi, l’urine et les excréments : «venez me voir, madame, mon mari a pris des photos »; "lorsque le matin les clients sortent la rue est vraiment impraticable : j'ai beau laver devant mon hôtel, le reste de la rue est répugnant. Pour mes enfants qui vont à l'école non plus, ce n'est pas bien qu'ils voient cela. Parfois, quand ce n'est pas leur jour, j'appelle la mairie, mais le temps qu'ils arrivent, c'est déjà tard. »

- les agressions sur personnes : plusieurs drames se sont déroulés au petit jour dans les rues d'Angers sur fond de licence généralisée. L'alcoolisation excessive peut donner lieu aussi à des relations sexuelles non voulues et non protégées, y compris dans le cadre de soirées étudiantes : on m’a rapporté, en assemblée générale de l'association Thiers-Boisnet, le suicide d'une jeune fille ayant été victime de viol en réunion, fait tragique confirmé par d'autres personnes dans un autre contexte.

Autrement dit, ce n'est pas tant le bruit en lui-même qui pose problème que ce qu'il fait entendre de la situation sociale relative de ceux qui font du bruit par rapport à ceux qui le subissent. Une vieille dame à laquelle j'essayais de faire dire que les responsables des cris la nuit n'étaient pas forcément contre elle ni contre les habitants m'a répliqué: "Alors comment expliquez-vous qu’ils aient défilé, ils étaient plus d'une centaine, samedi dernier à travers toute ma rue en hurlant "Angevins enculés"? "

C'est évidemment ici que le passage s'opère d'un objet technique et mesurable (en décibels, par exemple) à un objet sociologique renvoyant aux images que les uns ont des autres, et d'eux-mêmes, dans la cité dont ils partagent l'espace. Un espace qu'ils peuvent salir ou préserver, violenter ou prendre en charge. Et le glissement se poursuit, au fil des lectures exprimées du problème: on passe ainsi, au fil des réunions ou des rencontres (parfois en situation de mise en scène politique, parfois hors contexte), d'une analyse en termes d'incivilité liée aux nouveaux modes éducatifs aux conflits générationnels ; là, chacun brandit généralement son âge comme un bouclier. Puis les conflits sociaux s'expriment enfin, où les étudiants sont présentés comme de jeunes bourgeois, qui ont de l'argent, sont arrogants et pensent pouvoir s'affranchir des règles communes. Ils 
sont opposés dans les discussions aux pauvres gens, âgés ou travailleurs, dérangés dans leur sommeil, niés, délaissés par la police et sans autre alternative que de tomber malades à cause du bruit ou d'en devenir fous.

Car de quoi s'agit-il, au fond?

De conflits générationnels? «L'éducation a beaucoup changé depuis vingt ans et les jeunes ont pris l'habitude qu'on ne leur demande rien. Tout leur est dû, ils n'ont plus l'idée de l'effort et des autres. Des parents n'arrivent pas à se faire obéir de leur enfant de 4 ans : comment voulezvous que nous [habitants du quartier, membres du CCQ] leur demandions de respecter des règles quand ils ont 20 ans ? C'est cramé, on n'y arrivera plus. » $\mathrm{Ou}$ encore: "Je vis là depuis soixante-deux ans, dans cette rue j’y suis née, j’y ai eu toute ma vie, comme mes parents, avec mon frère, et j'aimerais bien y rester jusqu'à ma mort: je n'accepterai pas que des gamins qui n'en ont rien à faire, qui sont là pour deux ou trois ans d'études, m'obligent à partir. »

De conflits culturels? Anciens et nouveaux auraient un rapport différent à l'alcool : "pourtant nous aussi quand on était jeunes on aimait bien faire la fête»; «tout le monde aime bien boire un coup ou plus », « mais nous ça n'a jamais été comme ça, si souvent, si fort, et clairement pour emmerder le monde».

Ou de conflits de classe? «Les étudiants, ce sont des gens éduqués, moi si j’avais pu faire des études, j’aurais beaucoup aimé, mais à mon époque ce n'était pas possible. En tout cas, je n'aurais pas embêté les voisins ou les habitants »; «Les amendes ils s'en foutent: les étudiants se font des caisses pour régler les amendes. À cent vingt dans l'appartement et 45 euros l'amende, la mise à contribution est minimale »; « on loue pour faire des fêtes, on se cotise pour payer les amendes »; "Je ne suis pas d'Angers, j’ai acheté pour prendre ma retraite dans cette rue du centre-ville, l'agence m'a dit que c'était très bien; elle ne m'a rien dit sur les problèmes de bruit la nuit; or dans notre rue c'est invivable en fait, on regrette : où on ira maintenant, avec la crise et vu le quartier, notre appartement ne vaut plus rien ! ». «Si les étudiants peuvent le faire, pourquoi pas nous? » demandait un jeune travailleur issu des quartiers populaires pris à partie par des passants depuis la rue alors qu’il pendait sa crémaillère en centre-ville, avec musique et toutes fenêtres ouvertes.

C'est à ce point des écarts d'interprétation et des nœuds sociaux et générationnels du phénomène que l'analyse décalée et distanciée en termes de morphologie sociale apparaît très heuristique. De même que Jean-Claude Chamboredon parvenait à sortir d'une lecture psychologisante des difficultés quotidiennes dans les grands ensembles HLM pour décrire les heurts objectifs que produit la distance sociale dans la proximité spatiale ${ }^{18}$, il est 
possible de lire autrement la question du bruit à Angers quand on en considère la population.

\section{Le bruit du changement dans la morphologie sociale.}

Même si le fait est peu connu, et surtout peu reconnu par les habitants eux-mêmes, Angers se singularise aujourd'hui par sa jeunesse. Elle est en tête des villes du Grand Ouest pour la place des moins de 20 ans dans la population totale $(27 \%)$, nettement au-delà de la moyenne régionale $(25,6 \%)$ ou nationale $(24,8 \%)$. La pyramide des âges montre plus précisément que la jeunesse angevine va surtout de 15 à 29 ans, avec un effectif des 20 à 24 ans fortement surreprésenté, marqué par une particulière féminisation des effectifs ${ }^{19}$.

Mais lorsque l'on aborde la place de la jeunesse dans sa dynamique propre, on constate aussi que les jeunes de 15 à 24 ans représentent $40 \%$ du total des nouveaux arrivants à Angers entre 2001 et 2006, tandis que les seuls étudiants en constituent près d'un tiers. Ainsi, plus que toute autre catégorie de population, c'est la population estudiantine qui assure le renouvellement démographique de l'aire urbaine ${ }^{20}$.

Enfin, les étudiants ont largement investi la ville, où ils résident à $95 \%$ par rapport aux autres communes de l'agglomération, le plus souvent dans le parc locatif privé. En effet, selon une enquête de l'AURA (Agence d'urbanisme de la Région angevine) sur le logement étudiant dans la ville, ils ne seraient que $12 \%$ en résidences collectives ${ }^{21}$. Compte tenu du tiers résidant encore dans leur propre famille, cela signifie qu'à Angers ils sont massivement des locataires du parc privé (51\%), ce qui est un trait démographique spécifique à l'échelle nationale.

La carte de leur répartition dans la ville montre que l'immense majorité se concentre dans le parc locatif privé du centre-ville (42\%) et du péricentre $(30 \%)^{22}$, alors même que le plus gros pôle d'enseignement est le campus de Belle Beille, installé dans un quartier périphérique et regroupant plus du tiers des effectifs étudiants totaux. Mais cette polarisation dans le centre semble répondre à une nécessité autant qu'à un choix, car l'essentiel des T1 et T2 disponibles à la location à Angers y sont situés ${ }^{23}$. Alors que le centre-ville ne compte que $22 \%$ des résidences principales d'Angers, le parc privé y est plus dense qu'ailleurs, et les petits logements y sont surreprésentés ( $70 \%$ des logements privés à la location en centreville sont des T2 ou moins).

Ce quartier concentre en réalité $40 \%$ de l'offre de toute la ville en petits logements en location. Les étudiants y trouvent en outre tous les services de proximité, les lieux de distraction et de sorties (bars, restaurants, certaines 
boîtes de nuit) et des modes de transport desservant toutes les écoles et tous les sites universitaires. Et comme ils y trouvent statistiquement aussi plus fréquemment leurs amis, l'effet d'agglomération vient renforcer à son tour des choix individuels de localisation qui alimentent encore davantage la concentration dans le centre. Ainsi la place relative des étudiants dans le centre-ville d'Angers a-t-elle progressé tandis qu'ils occupaient peu à peu des logements plus grands (choisis en colocation, par cooptation le plus souvent) et délaissaient les «mauvais logements», mal équipés ou mal situés, du parc privé, au fil de leur carrière de locataires et d'une élévation de leurs exigences ${ }^{24}$. Or ce bouleversement de la démographie urbaine ne s'est pas traduit par un rajeunissement des symboles, de l'offre de services ou du discours de la ville sur elle-même. Il est étonnant de voir en effet à quel point cette dernière semble fonctionner encore comme si la population étudiante ne pesait rien ou presque: entre autres exemples, la première ligne du tracé du tramway ne dessert pas le premier pôle universitaire de la ville et le centre culturel officiel de l'Université d'État n'a ouvert qu'en 2006, sur les décombres d'une maison des étudiants associative qui n'a connu que quelques années d'activité ${ }^{25}$. Peut-on alors estimer qu'ouvrir ses fenêtres en grand toute la nuit pour diffuser aux alentours les échos d'une fête dans un appartement surpeuplé est une manière de se faire entendre, et, pendant du sentiment d'être niés des vieux Angevins, d'affirmer une présence collective d'autant plus vigoureusement qu'elle n'est pas véritablement reconnue par ailleurs?

Le bruit révèle sans doute la prise de possession nocturne de l'espace public par les étudiants, dont le poids croissant répond au développement de l'offre de formation supérieure à Angers et illustre localement le phénomène général de la massification de l'enseignement supérieur français et l'allongement de la durée des études. Il transcrit sur un mode sonore la transformation, favorisée par les dispositifs fiscaux, des logements du centre-ville en logements étudiants et se trouve concrètement amplifié par les nouveaux comportements d'alcoolisation excessive (des ivresses plus fréquentes et plus fortes) dans un contexte de malaise étudiant face à un avenir professionnel jugé sombre.

Mais cette transformation de la ville se lit à son tour au niveau de la mutation des vitrines de certains lieux du centre-ville, qui lui donne une physionomie particulière, en raison de l'effet d'aubaine pour les commerces de kebabs, pizzas et bars que constitue la concentration estudiantine. Sur les tensions sociales ressenties par les habitants victimes des nuisances se greffe un fort sentiment d'agacement envers les commerçants qui alimentent cette situation, en bénéficient financièrement et «poussent au crime». À la micro-échelle de la rue, on peut également observer entre les commerçants une lutte sourde, opposant ceux qui «profitent» des 
étudiants (bars, boîtes de nuit, épiceries ouvertes la nuit) et ceux qui s'en plaignent (pizzeria ne faisant pas d'affaires, boulangerie, pharmacie). Une économie politique du bruit la nuit pourrait éclairer les dynamiques à l'œuvre ${ }^{26}$. Mais l'essentiel semble néanmoins tenir à Angers dans la tension qui existe entre les changements démographiques objectifs et l'image stéréotypée et immuable de la ville, dont le CCQ se retrouve lui-même vecteur, parfois à son insu, et d'une manière qui l'empêche d'envisager de meilleures réponses et d'amener la mairie à aborder la question d'une manière plus féconde.

Le quotidien de l'élue novice que j'étais fut à la fois confus et intense, surchargé de sens, d'informations et de gestes à accomplir, le plus souvent dans l'urgence, à la lisière de l'impossible. Ce fonctionnement à flux tendu ne ménageait pas la possibilité d'un retour réflexif sur les pratiques, en raison de l'incessant basculement du rôle d'élue à celui d'enseignantchercheur, puis de maman culpabilisée de très jeunes enfants, avant de retourner à celui d'élue en réunions le soir. Ce retour ethnographique sur une expérience politique particulière ne doit donc pas donner l'illusion d'une histoire bien maîtrisée et d'une situation clairement appréhendée. La question du bruit à Angers a commencé à se poser longtemps avant mon arrivée et elle poursuit sa course aujourd'hui. Mais mon expérience permet de rendre compte, au plus près d'un terrain particulier, de la manière dont un certain type de bruit s'incarne dans cette ville, ce qu'il signifie et comment il peut devenir, ou non, une catégorie du débat politique, voire de l'intervention publique.

Si l'on peut considérer que «les luttes politiques sont dominées par la compétition permanente pour l'imposition du sens légitime à donner à l'action conduite ${ }^{27}$ », on a montré ici qu'il y a aussi en politique à conférer du sens à une action non conduite. C'est ce à quoi une sociologie compréhensive peut aboutir, mettant en lumière de l'intérieur les processus et les conflits par lesquels la décision politique d'une certaine forme d'inaction a pu dominer. Mais la compréhension du phénomène du bruit requiert aussi d'articuler le niveau de l'analyse ethnographique aux enseignements d'une vue macro-sociale de la ville et de ses transformations qui, à Angers en particulier, échappe trop à la connaissance commune.

Enfin, les conflits autour du bruit au sein du CCQ éclairent également les malentendus de la démocratie participative, où les habitants sont invités à venir s'exprimer sur les problèmes et les questions qui les concernent, devant des élus censés les écouter et tenir compte de leurs dires. Cette mise 
en scène néglige le caractère hautement social, processuel et négocié de la transformation d'une question sociale en question politique : celle-ci relève d'une construction complexe - plus délicate lorsque les individus sont là pour parler en leur nom de problèmes personnels - qui va de la mise au jour d'enjeux publics à l'élaboration d'un agenda décisionnel, via la construction de catégories de l'action politique ${ }^{28}$.

Le décalage entre la théorie de la démocratie participative et ce dont les élus sont capables dans les faits en termes de «mise en politique», les écarts entre les conceptions sous-jacentes des uns et des autres des modalités de l'action politique (discipline vs sécurité), et les retards entre les changements de la morphologie sociale d'une ville et leur perception par les habitants illustrent toutes les dimensions de ce malentendu. L'impossibilité de la résolution simultanée de ces hiatus explique pourquoi la mobilisation des habitants contre le bruit ne pouvait en l'occurrence que se traduire par une manière d'échec de tous les acteurs en présence ${ }^{29}$.

\author{
Elsa LAFAYE DE MicheaUX \\ elsa.lafaye-demicheaux@univ-rennes2.fr \\ MCF, Université de Rennes 2 \\ Centre d'économie de la Sorbonne
}

\begin{abstract}
NOTES
1. Julien Gracq, La Forme d'une ville (1985), Paris, Gallimard, 1995, p. 777.

2. Ibid., p. 778.

3. Par exemple dans le classement proposé chaque année depuis 2002 par le magazine Le Point.

4. Voir l'article de Philippe Le Guern dans ce numéro.

5. J'ai occupé la position d'adjointe au maire pour le quartier centre-ville de mars 2008 à mars 2010, au sein d'une équipe dirigée par le même maire, socialiste, depuis 2001 (et conseiller municipal depuis 1976). «Adjoint de quartier» est une fonction politique introduite alors à Angers, dans le prolongement de la mise en place de la loi Vaillant relative à la démocratie de proximité (loi no 2002-276 du 27 février 2002) et instituant les conseils de quartier pour les villes de 20000 habitants ou plus. Lors du précédent mandat, cette nouvelle loi s'était traduite par la création d'un service dédié à la participation des habitants au sein de la mairie et employant un certain nombre d'agents afin d'organiser et de suivre les dix conseils de quartier (dits «conseils consultatifs de quartier », CCQ). En 2008, la nouvelle majorité a choisi d'organiser le pouvoir municipal différemment, en cherchant à croiser les logiques thématiques et territoriales : ainsi, dix adjoints de quartier sont venus rejoindre les dix-huit adjoints thématiques aux fonctions établies de longue date (voirie, culture, enfance, jeunesse, sport, urbanisme, solidarités, commerce, etc.).

6. «La population, dans son ensemble et ses parties, avec ses formes, sa structure, qui ne s'expliquent que par sa nature propre; tel est l'objet de la morphologie sociale stricto sensu. C'est à elle que nous sommes ramenés toutes les fois que nous étudions les morphologies particulières. Ainsi il y a des faits de population, qu'on peut séparer de tout le reste, et rattacher les uns aux
\end{abstract}




\section{Elsa Lafaye de Micheaux}

autres sans sortir du domaine qu'ils constituent» (Maurice Halbwachs, Morphologie sociale, Paris, Armand Colin, 1938, p. 42).

7. Quotidien régional diffusé à 100000 exemplaires avec cinq éditions locales, le Courrier de l'Ouest (autrefois Courrier d'Angers) est d'abord un journal d'abonnés, au public plutôt âgé. Scruté par le cabinet du maire et lu par toute la municipalité, qu'on y abonne d'office, il est l'un des acteurs du conflit sur le bruit à travers sa rubrique "Courrier des lecteurs ».

8. Totalement novice en politique, de plus non affiliée au parti socialiste, je me voyais attribuer un mandat d'adjoint territorial lui-même en cours de définition.

9. Il s'agit à l'évidence de conflits d'usage entre les fonctions domestique, économique et récréative, voire patrimoniale, du quartier ; voir Thierry Kirat et André Torre (dir.), Territoires de conflits. Analyses des mutations de l'occupation de l'espace, Paris, L'Harmattan, 2008.

10. Autrement dit, une commande que la mairie adresse au CCQ : celui-ci forme alors un groupe de travail qui se charge, au fur et à mesure des réunions, de rendre une réponse sur le sujet.

11. Sous-titré «Trop d'alcool / trop de bruit tue la fête » dans une double optique de prévention de l'alcoolisme et de promotion de la tranquillité publique, novembre 2008.

12. «Je suis enceinte, fatiguée et je n’ai pas droit au sommeil », Courrier de l'Ouest, 2 juin 2009.

13. L'auteur de l'article a demandé au couple de lui faire suivre les réactions qui s'adressaient à lui directement (le journal avait publié sa photo, son nom et celle de son entrée d'immeuble), en particulier celles de la mairie.

14. Au passage, cette situation montre bien la valeur heuristique de l'approche compréhensive en sociologie lorsqu'il s'agit de dégager les façons et les raisons d'agir respectives des différents acteurs dans des contextes spécifiques. On peut trouver une bonne présentation théorique des vertus de la compréhension dans Cyril Lemieux, Le Devoir et la Grâce, Paris, Economica, 2009.

15. Michel Foucault, Sécurité, Territoire, Population. Cours au Collège de France 1977-1978, Paris, Hautes Études / Gallimard / Seuil, 2004.

16. $58,5 \%$ des 32000 étudiants angevins étaient en 2007 en niveau licence. Source: AURA (Agence d'urbanisme de la Région angevine), Éléments de connaissance du logement étudiant. Situation 2008, 2008, p. 11.

17. La moitié seulement des étudiants angevins sont du département et les deux tiers ne résident pas chez leurs parents, proportion plus élevée que pour la moyenne nationale. Source : ibid., p. 14.

18. Pour lui, ce sont les caractéristiques morphologiques de cette population (en particulier la pyramide des âges et la dynamique de peuplement) qui sont la clé de l'analyse des transformations sociales dans les grands ensembles. En effet, «les divisions sociales $[y]$ sont surdéterminées par les conditions de constitution de cette population et par les particularités de sa composition » (JeanClaude Chamboredon, "Proximité spatiale et distance sociale des grands ensembles et leur peuplement », Revue française de sociologie, vol. XI, 1970, p. 3-33).

19. Les tranches de population d'amplitude de 5 ans comptent en moyenne autour de $2 \times 4000$ individus (hommes et femmes), sauf pour les catégories d'âge les plus élevées, où le chiffre baisse après 70 ans. Mais trois tranches d'âges se distinguent du profil d'ensemble: les 15-19 ans (7 000 garçons/8000 filles environ) et les 25-29 ans $(2 \times 6500)$ d'une part, les 20-24 ans surtout, avec plus de 9000 garçons et plus de 11000 filles en 2006, soit une catégorie d'âge plus que deux fois surreprésentée par rapport aux autres. Source: AURA, La Jeunesse du territoire angevin, 2010.

20. Alors que les étudiants représentent $23 \%$ des nouveaux arrivants à Nantes et Brest, ils en constituent 29 \% à Angers et Rennes selon l'AURA (Quivient s'installer dans le Grand Ouest?, avril 2011).

21. Le logement en cité universitaire ne correspond qu'à $6 \%$ du total, les foyers de jeunes travailleurs à $2 \%$ (AURA, Éléments de connaissance du logement étudiant. Situation 2008, op. cit., p. 14).

22. Pour ce qui concerne les seuls allocataires d'aides au logement (données de la CAF citées in ibid., p. 16).

23. Données INSEE citées in ibid., p. 17. Ici intervient une subtilité participant à la difficulté récurrente de mobiliser des informations statistiques robustes à des fins d'explication des enjeux auprès des habitants réunis en CCQ : les contours politiques du quartier dont j'étais l'élue, dit quartier "centre-ville, La Fayette, Éblé », sont différents des contours administratifs et statistiques définis par l'INSEE dans le cadre de ses analyses infra-communales dites «aux IRIS ». Au-delà de ces différences de périmètre, c'est bien plus largement le recours à des travaux scientifiques pour éclairer les questions du quotidien qui manque au niveau des élus de quartier : les services munici- 
paux (en l'occurrence, la prospective) se sont révélés très défiants à l’idée de laisser entre nos mains des données et des études dont ils ne contrôleraient pas l'emploi que l'on pourrait en faire. Ainsi, on me refusait l'utilisation de données sur les revenus de la population du centre-ville de peur que je ne me trompe dans les explications relatives à la médiane et la moyenne. J'ai dû mettre en avant une agrégation de sciences sociales pour avoir le droit de citer ces données en public, lors d'une des premières réunions du CCQ où je souhaitais porter à la connaissance des habitants la composition socio-démographique réelle du centre-ville, bien éloignée de l'image «bourgeoise » qu'il donne de lui-même.

24. AURA, Éléments de connaissance du logement étudiant. Situation 2008, op. cit., p. 24.

25. L'association Maison des étudiants d'Angers a vécu de 1997 à 2005. Cf. Anne Lafon, Fonds de l'association de la Maison des étudiants d'Angers (répertoire numérique détaillé), 2008, <http:// www.angers.fr/uploads/media/FRAC049007_47_J_-_Maison_des_Etudiants.pdf>.

26. Idée que Jean-François Bayart aborde brièvement dans sa chronique sur la «privatisation de l'espace public », Sociétés politiques comparées, no 19, 2009, <http://www.fasopo.org/reasopo/ n19/chronique.pdf>.

27. Philippe Braud, Sociologie politique, Paris, LGDJ, 2006, p. 545; cité par Paul Bacot, «Compte-rendu de lecture », Mots. Les langages du politique, n 84, 2007, p. 109.

28. Pierre Lascoumes et Patrick Le Galès, Sociologie de l'action publique, Paris, Armand Colin, 2010, p. 66-86.

29. Je tiens à remercier Samira Echahid pour l'érudition généreuse de sa relecture, en particulier pour la belle référence à Jean-Claude Chamboredon. J'ai aussi bénéficié des critiques très justes et toujours bienveillantes de Bénédicte Havard-Duclos et d'Emmanuelle Quiniou.

\section{RÉSUMÉ}

Le thème des nuisances sonores s'est rapidement imposé aux politiques publiques comme un enjeu du quotidien des habitants, notamment exprimé dans le cadre du développement d'instances participatives comme les conseils de quartier. Les habitants victimes de ces nuisances analysent ce phénomène en termes d'incivilités ou de détérioration du «vivre ensemble ». Cet article retrace, dans le cas d'Angers et à partir d'une enquête de terrain participative où l'auteur occupait la position singulière d'élue municipale, la mobilisation des différents acteurs concernés (associations, riverains, élus, étudiants) et son échec prévisible. En effet, la question du bruit semble moins s'expliquer par une sociologie des incivilités que traduire des évolutions de la morphologie sociale angevine.

\section{SUMMARY}

Noise pollution has rapidly emerged as a key public policy issue relevant for citizens everyday life and specifically revealed by the development of local-level participatory bodies such as neighborhood councils. Residents suffering from those nuisances interpret them either as marks of uncivil behaviour or as a weakening of the sense of community. The present article, focussing on the city of Angers and building on a participant observation carried out by the author holding the unusual position of town councillor, chronicles the mobilisation of several stakeholders (associations, tenants, elected officials, students) and its predictable failure. In fact, rather than being the instantiation of a "sociology of incivility", noise pollution designates a set of changes undergone by demographic, urban, and social structures. 